\title{
Electronic control over detachment of a self- doped water-soluble conjugated polyelectrolyte
}

\author{
Kristin M Persson, Roger Gabrielsson, Anurak Sawatdee, David Nilsson, Peter Konradsson \\ and Magnus Berggren
}

\section{Linköping University Post Print}

\section{Tweet}

N.B.: When citing this work, cite the original article.

Original Publication:

Kristin M Persson, Roger Gabrielsson, Anurak Sawatdee, David Nilsson, Peter Konradsson and Magnus Berggren, Electronic control over detachment of a self-doped water-soluble conjugated polyelectrolyte, 2014, Langmuir, (30), 21, 6257-6266.

http://dx.doi.org/10.1021/la500693d

Copyright: American Chemical Society http://pubs.acs.org/

Postprint available at: Linköping University Electronic Press http://urn.kb.se/resolve?urn=urn:nbn:se:liu:diva-106251 


\section{Electronic control over detachment of a self-doped}

\section{water-soluble conjugated polyelectrolyte}

Kristin M. Persson ${ }^{\dagger}$, Roger Gabrielsson ${ }^{\dagger,}$, Anurak Sawatdee ${ }^{\S}$, David Nilsson ${ }^{\S}$, Peter

Konradsson ${ }^{+}$, Magnus Berggren ${ }^{\dagger, *}$

$\dagger$ Department of Science and Technology, Linköping University, 60174 Norrköping, Sweden

¥Department of Physics, Chemistry and Biology, Linköping University, 58183 Linköping,

Sweden

$\S$ Department of Printed Electronics, Acreo Swedish ICT AB, 60117 Norrköping, Sweden

Water-soluble conducting polymers are of interest to enable more versatile processing in aqueous media as well as to facilitate interactions with biomolecules. Here, we report a substituted poly(3,4-ethylenedioxythiophene) derivative (PEDOT-S:H) that is fully water-soluble and selfdoped. When electrochemically oxidizing a PEDOT-S:H thin film, the film detaches from the under-laying electrode. The oxidation of PEDOT-S:H starts with an initial phase of swelling followed by cracking before it finally disrupts into small flakes and detaches from the electrode. We investigated the detachment mechanism and found that parameters such as the size, charge and concentration of ions in the electrolyte, the temperature and also the $\mathrm{pH}$ influence the characteristics of detachment. When oxidizing PEDOT-S:H, the positively charged polymer backbone is balanced by anions from the electrolyte solution and also by the sulphonate groups 
on the side chains (more self-doping). From our experiments, we conclude that detachment of the PEDOT-S:H film upon oxidation occurs in part due to swelling caused by an inflow of solvated anions and associated water, and in part due to chain rearrangements within the film, caused by more self-doping. We believe that PEDOT-S:H detachment can be of interest in a number of different applications, including addressed and active control of the release of materials such as biomolecules and cell cultures.

\section{Introduction}

During the last decades, conducting polymers have been increasingly explored for use in bioelectronics applications. ${ }^{1,2}$ Poly(3,4-ethylenedioxythiophene) (PEDOT) and polypyrrole (PPy) are two commonly utilized biocompatible ${ }^{3,4}$ materials, that have been used for drug release, ${ }^{5,6}$ stimulation of cells during growth and to transfer electronic signals to and from biological tissues. ${ }^{7,8}$ Due to its superior (bio-)stability ${ }^{9}$ and excellent conductivity ${ }^{10}$ PEDOT has become the prime choice in bioelectronics.

Fully water-soluble conducting polymers are of interest to enable more versatile processing methods and for use in combination with biomolecules. ${ }^{11-13}$ One route to achieve water-solubility in conducting polymers is to introduce charged side groups to the monomer. Such covalently bonded substituents may also make the resulting polymer self-doped, ${ }^{14}$ meaning that the required counter ion during doping is a side group of the polymer chain itself. Since the first watersoluble conducting polymers ${ }^{14,15}$ were reported, water-soluble PEDOT derivatives have been enabled for example through poly(4-(2,3-dihydrothieno[3,4-b]-[1,4]dioxin-2-yl-methoxy)-1butanesulfonic acid, sodium salt (PEDOT-S). ${ }^{16-19}$

We recently demonstrated human epithelial cell detachment using a PEDOT-S:H thin film. ${ }^{20}$ In PEDOT-S: $\mathrm{H}, \mathrm{Na}^{+}$has during synthesis been replaced by $\mathrm{H}^{+}$(or rather $\mathrm{H}_{3} \mathrm{O}^{+}$) as the charge 
compensating ion to the sulphonate groups. When electrochemically oxidized, PEDOT-S:H swells, cracks and detaches (figure 1), taking cultured cells along with it. Adherent cells are commonly detached with enzymatic methods that however may damage the surface proteins on the cells ${ }^{21}$ creating a need for alternative methods. Cells detached with PEDOT-S:H showed improved preservation of surface proteins compared to cells detached using enzymatic methods. Further, re-seeded cells assumed a normal morphology. ${ }^{20}$ In this paper, we have characterized the mechanism of PEDOT-S:H detachment in detail. We believe that thin film materials that actively disrupts and detaches upon electrochemical oxidation could also be used in other applications in which precise control over release is of interest, such as in drug delivery and as smart glues for packaging and labels. ${ }^{22}$

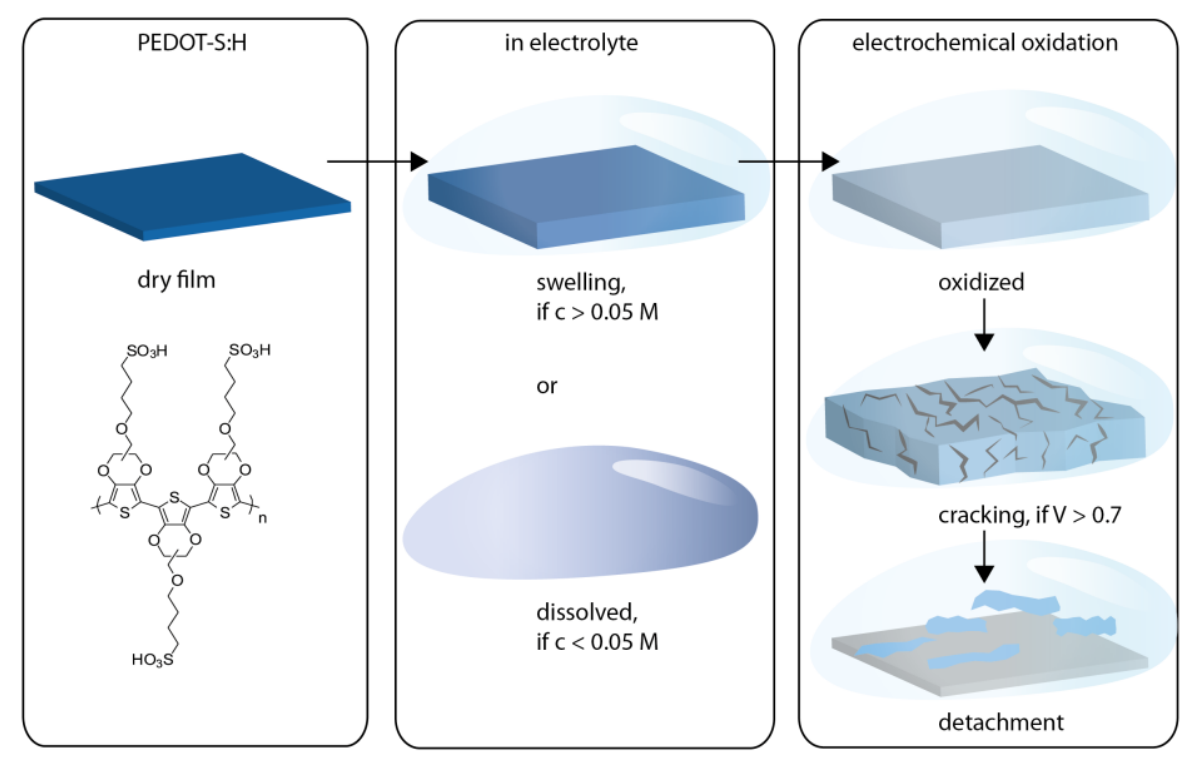

Figure 1. When a thin film of PEDOT-S:H (left panel) is submerged in aqueous electrolyte (middle panel) it will swell or, if $\mathrm{c}<0.05 \mathrm{M}$, dissolve. If $\mathrm{c}>0.05 \mathrm{M}$, the polymer film will not dissolve and can be electrochemically oxidized in an aqueous electrolyte (right panel) In some electrolytes it will start to crack followed by subsequent disintegration to smaller flakes followed by detachment. 
Earlier work on dissolution or delamination of conducting polymers has focused on the prevention of uncontrolled delamination through improved adhesion characteristics. ${ }^{23}$ Another example involves dissolution of polythiophene films in organic solvents due to oxidative degradation. ${ }^{24}$ We have not found any previous examples of electronic control of detachment of a conducting polymer that operates in water.

During synthesis PEDOT and PPy will be p-doped (oxidized), ${ }^{25,26}$ and to maintain overall electroneutrality anions will be incorporated in the film. During subsequent electrochemical redox switching in solution, changes in conductivity, colour and volume will occur. Polymer swelling during redox switching has been studied extensively in $\mathrm{PPy}^{27-30}$ and to some extent also in PEDOT $^{31,32}$ and employed in actuator applications. ${ }^{33-35}$ The direction of ion and solvent movement, and thus volume change, upon an applied potential will depend on the fixed charges within the (oxidized) polymer $\left(\mathrm{P}^{+}\right.$in equation 1$)$. If small anions $\left(\mathrm{A}^{-}\right)$are used as the counter ions during synthesis, these can leave the film (represented by brackets) upon reduction to a neutral state $\left(\mathrm{P}^{0}\right)$. If instead large anions, for example polyanions, $\left(\mathrm{M}^{-}\right)$serve as counter ions, these will remain within the film upon reduction and cations from the surrounding electrolyte will enter the film (equation 2). ${ }^{36}$ If the films are re-oxidized, anions will either enter the film (equation 1) or be expelled (equation 2).

$\left[\mathrm{P}^{\mathrm{n}+}: \mathrm{A}^{\mathrm{n}-}\right]+\mathrm{ne}^{-} \rightarrow\left[\mathrm{P}^{0}\right]+\mathrm{nA}^{-}+\mathrm{nC}^{+}$

$\left[\mathrm{P}^{\mathrm{n}+}: \mathrm{M}^{\mathrm{n}-}\right]+\mathrm{ne}^{-}+\mathrm{nC}^{+} \rightarrow\left[\mathrm{P}^{0}: \mathrm{M}^{\mathrm{n}-}: \mathrm{C}^{\mathrm{n}+}\right]$

In the as-synthesized state of PEDOT-S:H the sulphonate groups will balance the positive charges on the polymer backbone, thus making the system intra-molecularly self-doped. The degree of doping has been estimated, using both elementary analysis and titration, to 0.3 in PEDOT-S:H. ${ }^{13,18}$ Thus, there will be an excess of fixed negative charges, balanced by protons, within the polymer film as all sulphonate groups are not involved in self-doping. When oxidizing 
a self-doped polymer further from its as-synthesized state, the increased number of positive charges on the polymer can either be balanced by the sulphonate groups on the polymer itself, by anions from the electrolyte or by a combination of the two processes. Previously, it has been reported that an increased degree of self-doping is the dominating process during redoxswitching in these polymer systems. ${ }^{14,16}$

Here, we report on the mechanism of swelling and subsequent detachment of the PEDOT-S:H films during electrochemical oxidation and how the detachment characteristics are influenced by the electrolyte used, its concentration, its $\mathrm{pH}$ level and temperature. In addition, atomic force microscopy (AFM) and electrochemical quartz crystal microbalance with dissipation (EQCM-D) studies have been utilized to investigate and monitor the detachment mechanism. EQCM is used to study small changes in mass depending on the applied voltage. ${ }^{37,38}$ In QCM-D measurements the frequency response reflects the viscoelastic properties, thickness and density of any mass coupled to the quartz crystal that will appear as a change in frequency $(f)$ and dissipation $(D)$ at various overtones. ${ }^{39-43}$ The EQCM-D senses the mass of the deposited film as well as trapping of water and co-ions. With appropriate modeling of the $f$ and $D$ data the thickness and viscosity for the adsorbed or deposited layer can be estimated. For further information about EQCM-D, please refer to the supplementary information.

\section{Experimental section}

Polymer synthesis. PEDOT-S:H was synthesized according to a previously published protocol. ${ }^{20}$

Electrolytes. $\mathrm{NaCl}, \mathrm{Na}_{2} \mathrm{SO}_{4}, \mathrm{CaCl}_{2}, \mathrm{MgCl}_{2}, \mathrm{FeCl}_{3}$, Dodecylbenzene sulfonic acid sodium salt (NaDBS), $\mathrm{HCl}$ (37\%), $\mathrm{NaOH}$ and polydimethyldiallylammonium chloride (pDADMAC), 20\% 
solution, were purchased from Sigma. Poly(vinylphosphonic acid) (PVPA), 30\% solution and poly(acrylic acid) (PAA), 35\% solution, was purchased from Rhodia.

Polymer coating. PEDOT-S:H $(20 \mathrm{mg} / \mathrm{ml})$ was bar coated on PEDOT:PSS foil (Agfa-Gevaert Orgacon F-350) that had been washed in acetone and water and then treated with ozone. PEDOT-S:H was coated using a BYK Gardner bar-coater at speed $100 \mathrm{~mm} / \mathrm{s}$. After coating, the polymer film was dried at $80^{\circ} \mathrm{C}$ for 45 minutes. The thickness of the resulting film was measured using a surface profiler (Dektak 3 ST, Veeco).

Stability experiments. Bar coated PEDOT-S:H on PEDOT:PSS foil was used. Substrates approximately $2.5 \mathrm{~cm}^{2}$ in size were submerged in electrolyte and moved back and forth. $\mathrm{NaCl}$ electrolytes were used and the concentrations were varied from $0.01 \mathrm{M}$ to $2.0 \mathrm{M}$. For other electrolytes a concentration of $0.1 \mathrm{M}$ was used. All solutions were prepared in water. For pDADMAC, PVPA and PAA the concentrations were calculated for monomer units.

Detachment experiments. A potential of $1.0 \mathrm{~V}$ vs. $\mathrm{Ag} / \mathrm{AgCl}$ was applied, using a potentiostat ( $\mu$ Autolab, EcoChemie), between the bar coated PEDOT-S:H substrate $\left(2.5 \mathrm{~cm}^{2}\right.$ in size) and a platinum counter electrode, placed in a plastic beaker filled with the electrolyte to be investigated. For full details, see supporting information.

Cyclic voltammetry (CV). Bar coated PEDOT-S:H was used for cyclic voltammetry in three different electrolytes; $\mathrm{NaCl}$, PVPA and pDADMAC, at concentrations of $0.1 \mathrm{M}$. Three subsequent $\mathrm{CV}$ scans from $0 \mathrm{~V}$ to $+0.5 \mathrm{~V}$ to $-1.0 \mathrm{~V}$ vs. $\mathrm{Ag} / \mathrm{AgCl}$ were recorded at a scan speed of $0.02 \mathrm{~V} / \mathrm{s}$. A platinum counter electrode was used. The second scan was used for evaluation and comparisons.

Electrochemical Quartz Crystal Micro Balance with Dissipation (EQCM-D). All electrochemical QCM-D measurements were performed using a Q-Sense AB (QEM 401) 
electrochemistry module connected to a Q-Sense (E4) chamber and an Autolab PGSTAT 10 was used as the potentiostat setup. Quartz crystals were analyzed and the coated with PEDOT-S:H. After obtaining a baseline in air, the dry mass could be calculated using the Sauerbrey relation. When performing meausurements in electrolyte, the Sauerbrey relation in combination with the Voigt model was used to evaluate the viscoelastic properties of the film. For full details, see supporting information.

Atomic Force Microscopy (AFM). Four different samples of PEDOT-S:H on ITO on glass were analysed with AFM; including one pristine, two incubated in $0.1 \mathrm{M} \mathrm{NaCl}$ and one oxidized (at $0.9 \mathrm{~V}$ in $0.1 \mathrm{M} \mathrm{NaCl}$ ) films. The films were dipped into water to remove residual salt. After drying tapping mode AFM (Veeco Dimension 3100 was carried out in air at room temperature. For full details, see supporting information.

Patterning and inkjet printing. PEDOT:PSS foil (Agfa-Gevaert Orgacon F-350) was patterned using standard lithography techniques and reactive ion etching. The patterned PEDOT:PSS electrode substrates were coated with PEDOT-S:H $(5 \mathrm{mg} / \mathrm{ml})$ using an inkjet printer (Dimatix 2831). The electrodes of PEDOT:PSS were coated with PEDOT-S:H by printing an areal rectangle covering all electrodes. To define the electrolyte area, SU-8 was paintbrushed to allow only the area coated with PEDOT-S:H to be exposed to electrolyte. For full details, see supporting information.

\section{Results and Discussion}

Polymer characteristics. We have previously characterized the synthesis, composition and conductivity of PEDOT-S:H in detail. ${ }^{18,20}$ The material exhibit a high degree of self-doping and a conductivity of $30 \mathrm{~S} \mathrm{~cm}^{-1}$ in the oxidized state. In the neutral state, the conductivity decreases significantly, a fact that has been employed in electrochemical transistors. ${ }^{11}$ 
PEDOT-S:H was successfully deposited using spin- and bar coating and also inkjet printing, on top of conducting substrates such as PEDOT:PSS foil and ITO on glass. AFM studies of the film revealed that spin coated pristine films of PEDOT-S:H were relatively smooth with a low degree of roughness (figure 2a). Bar coated films cut into $2.5 \mathrm{~cm}^{2}$ pieces were used in most experiments. The thickness of these films was $0.5 \mu \mathrm{m}$. The estimated density of PEDOT-S:H is $1.1 \mathrm{~g} / \mathrm{cm}^{3}$ which then corresponds to $137.5 \mu \mathrm{g}$ for the $0.5 \mu \mathrm{m} * 2.5 \mathrm{~cm}^{2}$ films.
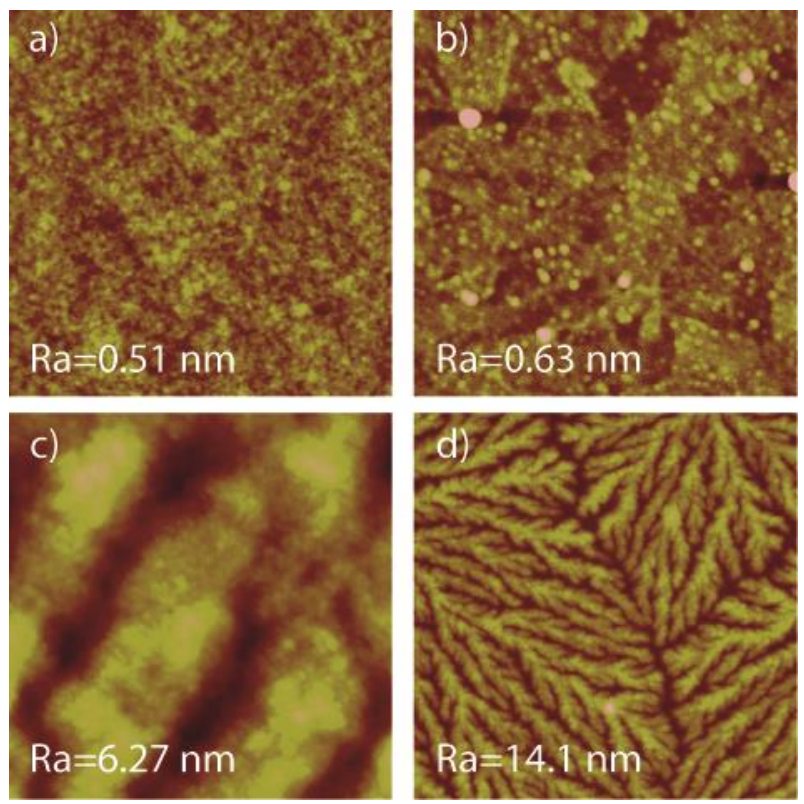

Figure 2. Tapping-mode AFM images of dry films, a) PEDOT-S:H, b) PEDOT-S:H incubated $90 \mathrm{~s}$ in $0.1 \mathrm{M} \mathrm{NaCl}, \mathrm{c}$ ) and d) PEDOT-S:H oxidized $90 \mathrm{~s}$ in $0.1 \mathrm{M} \mathrm{NaCl}$. Scan sizes are $1 \mu \mathrm{m}$ in a)-c) and $10 \mu \mathrm{m}$ in d). The height scale is $8 \mathrm{~nm}$ in a) and b), $50 \mathrm{~nm}$ in c) and $150 \mathrm{~nm}$ in d). Also shown are calculated roughness $(\mathrm{Ra})$ values.

PEDOT-S:H in electrolyte. The stability of bar coated PEDOT-S:H films on top of PEDOT:PSS in various electrolytes without an applied potential was evaluated by submerging them in solution. The induced changes in the PEDOT-S:H film upon incubation in electrolyte were characterized using AFM. After 90 seconds the polymer appeared swollen (figure 2b) and 
the calculated roughness had increased compared to the pristine film. This confirmed that electrolyte will enter the film regardless of any applied potential and thus contribute to polymer swelling, as previously shown for PPy. ${ }^{30}$ Swelling was also monitored using EQCM-D. An immediate decrease of the frequency indicated an increase in mass (figure 3). Moreover, an increase in dissipation appeared after exposure to electrolyte, indicating changes in the viscoelastic properties of the film, i.e. swelling due to inflow of hydrated ions.

Stability in different electrolytes. Next, we investigated the stability in different electrolytes and concentrations. In deionized water, PEDOT-S:H films were spontaneously and quickly dissolved. We assumed that there would be a limit for how much water the film could incorporate without becoming spontaneously dissolved. Using $\mathrm{NaCl}$ as the electrolyte, the stability of PEDOT-S:H films were tested at different concentrations (from $0.01 \mathrm{M}$ to $2.0 \mathrm{M}$ ). We found that for concentrations lower than $0.05 \mathrm{M}$ the polymer film was dissolved (figure 1), as the inflow of water into the film was sufficiently large to dissolve PEDOT-S:H completely.

The stability was further tested in different electrolyte solutions, all at a concentration of 0.1 M. The impact on swelling and dissolving was studied for different divalent cations and anions $\left(\mathrm{CaCl}_{2}\right.$ and $\left.\mathrm{Na}_{2} \mathrm{SO}_{4}\right)$ as well as polycations and polyanions (pDADMAC and PVPA). Supporting figure S1 shows the suggested ion movements for different electrolytes. As long as the concentration was higher than $0.05 \mathrm{M}$ we found that the type of ions in the electrolyte did not influence the stability and the films were stable for at least four weeks (longer times not tested). The influence of $\mathrm{pH}$ on stability was first investigated using diluted aqueous $\mathrm{HCl}$ and $\mathrm{NaOH}$ solutions. Only for pH values below 1.3 or above 12.7 did the PEDOT-S:H films remain stable. These values correspond to electrolyte concentrations higher than $0.05 \mathrm{M}$. If instead adding $\mathrm{HCl}$ or $\mathrm{NaOH}$ to a $0.1 \mathrm{M} \mathrm{NaCl}$ solution the $\mathrm{pH}$ did not influence the stability characteristics. 

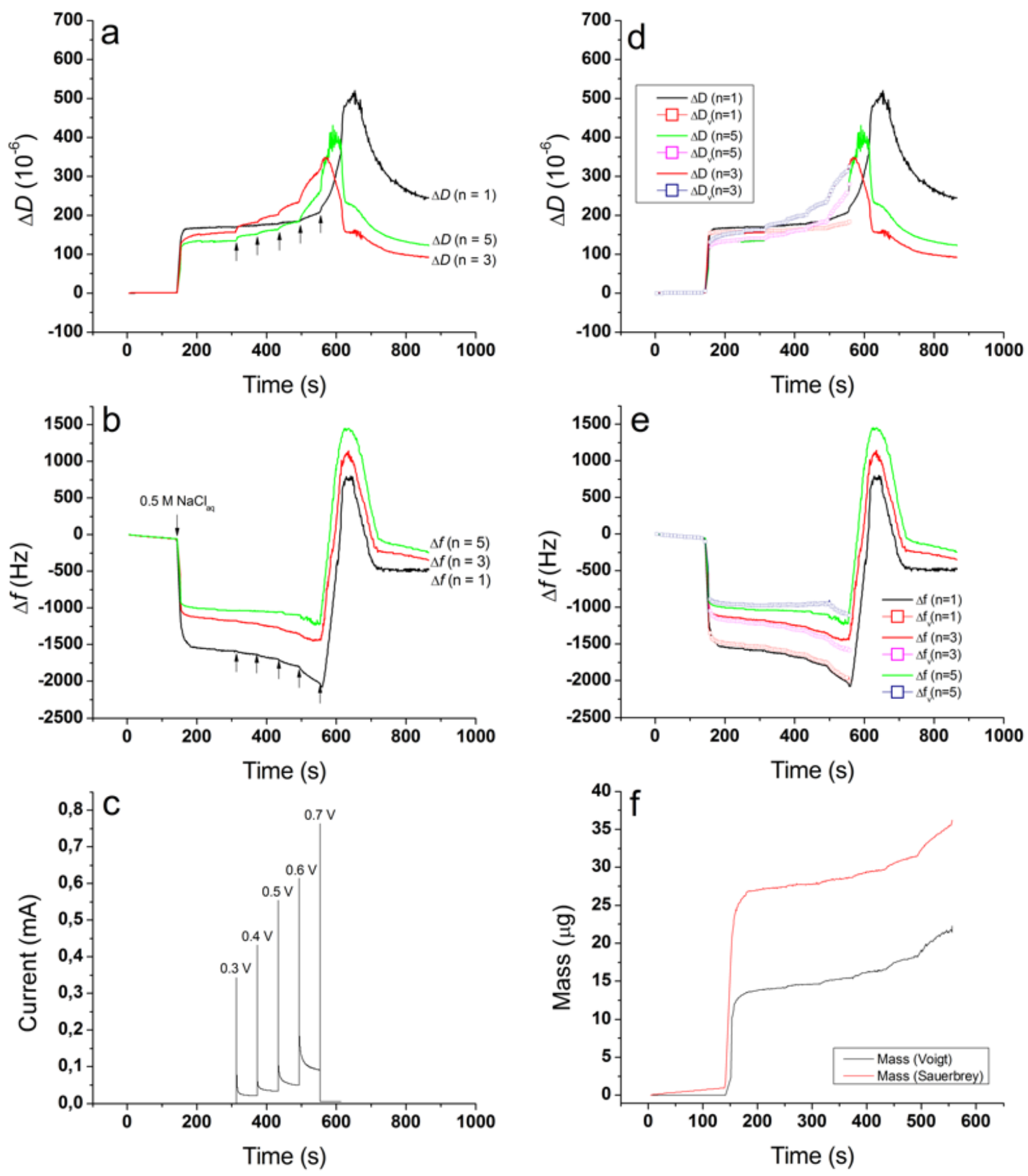

Figure 3. Time resolved EQCM-D experimental data for $\Delta D$ (a) and $\Delta f$ (b) respectively versus time time at $\mathrm{n}=1$ (black lines), $\mathrm{n}=3$ (red lines) and $\mathrm{n}=5$ (green lines) for the EQCM-D experimental data and the best fit between the Voigt model (obtained using Q-Soft (Q-Sense AB)) 
and the experimental data are shown in (d) for $\Delta \mathrm{D}$ and (e) for $\Delta f$ respectively as red open squares for $\left(\Delta f_{\mathrm{n}=1}\right.$ and $\left.\Delta \mathrm{D}_{\mathrm{n}=1}\right)$, pink open squares for $\left(\Delta f_{\mathrm{n}=3}\right.$ and $\left.\Delta \mathrm{D}_{\mathrm{n}=3}\right)$ and blue open squares for $\left(\Delta f_{\mathrm{n}=5}\right.$ and $\Delta \mathrm{D}_{\mathrm{n}=5}$ ). The black arrows in figure $3 \mathrm{a}, \mathrm{b}$ indicate the potential stepping, going from $0.3 \mathrm{~V}$ to $0.7 \mathrm{~V}$, from the illustrated amperogram (c). The model output obtained from the best fit is illustrated in (f) for the Voigt estimated mass (black line) and Sauerbrey related mass (red line).

In basic electrolytes, the polymer backbone will be undoped, as evidenced by the dark blue/purple colour of the PEDOT-S:H film, which results from absorption by the $\pi-\pi^{*}$ transition at 500-600 nm. ${ }^{12}$ This means that the butane-sulphonate side chains of PEDOT-S:H will no longer be needed for charge compensation and will therefore be strongly dissociated, both interand intra-molecularly due to the excess negative charge present.
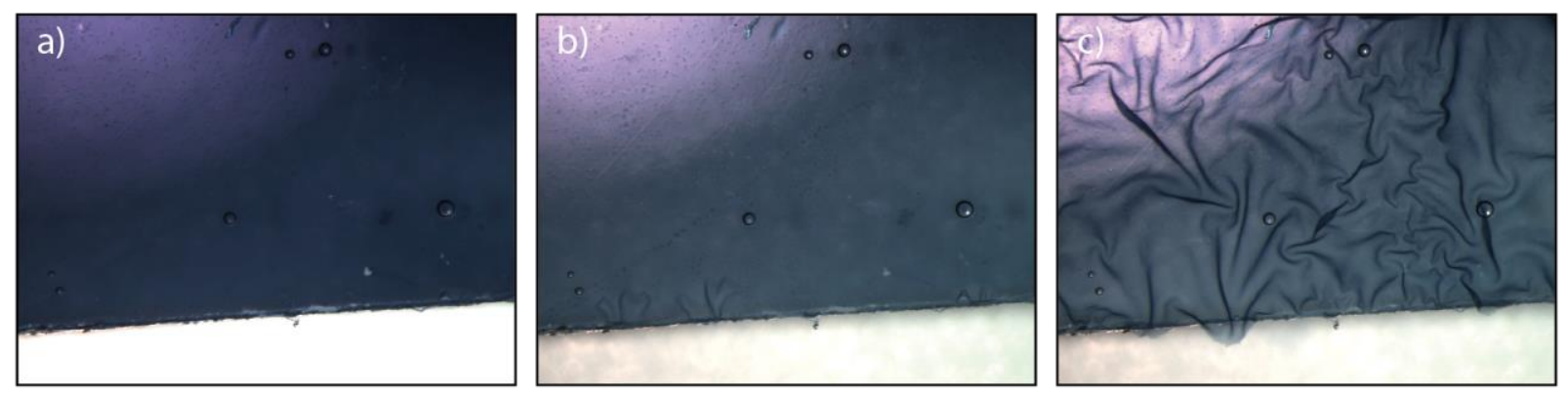

Figure 4. Optical microscope images of the detachment of a bar coated PEDOT-S:H film. In a) the film before oxidation is shown. In b) the film is oxidized and has started to fold in the lower left corner. In c) the film is swollen and extensively folded.

Detachment of PEDOT-S:H. The detachment process upon oxidation at potentials above $+0.7 \mathrm{~V}$ vs. $\mathrm{Ag} / \mathrm{AgCl}$ (figure 4) occurs in different steps characterised by an initial swelling phase, then cracking and folding of the PEDOT-S:H film until it finally disintegrates into small flakes. ${ }^{20}$ Gentle agitation of the electrolyte will completely remove the film from the underlying substrate. AFM analysis revealed that, upon oxidation, the morphology of the PEDOT-S:H film was 
markedly changed. Figure $2 \mathrm{c}$ and $\mathrm{d}$ show a folded structure indicating swelling and cracking of the film. These changes were also evident from the large differences in surface roughness. If instead reducing the polymer to its neutral state, no additional swelling or cracking occurred (data not shown). Using absorption spectroscopy, we have not detected any dissolved PEDOT$\mathrm{S}: \mathrm{H}$ in the electrolyte after detachment (data not shown), the detached polymer film is only present as small flakes.

As discussed above, during oxidation additional positive charges along the polymer chain can be compensated for either by anions from the electrolyte, or by rearrangement of the polymer chains to increase the degree of self-doping. ${ }^{14,16}$ As we see swelling upon oxidation, it is likely that anions enter the polymer in combination with additional self-doping. The suggested ion movements during oxidation are summarized in figure S1c.

It is clear that swelling is an important part of the detachment mechanism. Already when submerged in electrolyte the polymer film will take up water and expand. The swelling will increase further during oxidation, as indicated by a mass increase in the EQCM-D experiments (figure 3). Ion diffusion from the electrolyte for charge compensation during polymer oxidation will be accompanied by solvent, as has been shown for PPy actuators. ${ }^{30,44}$ When applying an electric potential between the substrate and the counter electrode ions will also initially migrate in the resulting electric field. ${ }^{45}$ Swelling during redox reactions will also be influenced by differences in ion concentrations and thus water activities between the electrolyte and the polymer film, leading to variations in osmotic expansion. ${ }^{46,47}$

Detachment in different electrolytes. To evaluate the impact of the types of ions on the detachment process, different electrolytes were tested. First we compared detachment in $0.1 \mathrm{M}$ aqueous electrolytes including $\mathrm{NaCl}, \mathrm{NaDBS}, \mathrm{Na}_{2} \mathrm{SO}_{4}, \mathrm{CaCl}_{2}, \mathrm{FeCl}_{3}$ and $\mathrm{MgCl}_{2}$. The 
electroactivity of PEDOT-S:H in the various electrolytes was confirmed from the fact that all the films exhibited an electrochromic colour change during cyclic voltammetry. In $\mathrm{NaCl}, \mathrm{NaDBS}$ and $\mathrm{Na}_{2} \mathrm{SO}_{4}$ PEDOT-S:H detached, showing that neither the size nor the charge of the anion influences the detachment mechanism significantly. In the case of cations the charge influenced the detachment process and in the case of $\mathrm{CaCl}_{2}, \mathrm{FeCl}_{3}$ and $\mathrm{MgCl}_{2}$ the films could not be detached at all. We assume that di- or tri-valent ions will enter the polymer film and cross-link the polymer chains at the sites of the sulphonate groups ${ }^{48}$ (figure S1c), assumingly preventing further self-doping upon oxidation Further, using EQCM-D, we observed less swelling of the polymer film when using electrolytes with di- or trivalent cations (data not shown), as also noted previously for PPy actuators. ${ }^{27,48}$

To further investigate the cross-linking, we performed a combined experiment; when first incubating a PEDOT-S:H film in $\mathrm{CaCl}_{2}$ and then transferring it to $\mathrm{NaCl}$ before electrochemical oxidiation, the film detached. This indicates that the $\mathrm{Ca}^{2+}$ ions had been exchanged by $\mathrm{Na}^{+}$ions. However, for incubation times in $\mathrm{CaCl}_{2}$ longer than 3 days, the detachment time increased (figure 5a). After seven days of incubation it was in fact hard to achieve detachment at all. We have previously shown that when PEDOT-S:H films were incubated in $\mathrm{NaCl}$ for extended period of times the detachment time was not affected to the same extent. ${ }^{20}$ When adding $\mathrm{CaCl}_{2}$ to $\mathrm{NaCl}$ the detachment time increased with increasing amount of $\mathrm{CaCl}_{2}$ added (figure 5b), indicating that there is a certain upper limit of the degree of cross-linking for which detachment does not occur.

When increasing the electrolyte concentration the detachment time increased for all tested electrolytes (figure 6a). At concentrations above $1.0 \mathrm{M}$, typically only fractions of the polymer films detached. When increasing the concentration of the electrolyte the difference in water activity between the polymer film and the electrolyte will decrease. The tendency of water to 
enter the film (osmatic expansion) will then decrease, in turn decreasing the swelling of the film. A similar swelling behaviour has been observed for PPy films. ${ }^{46,49}$

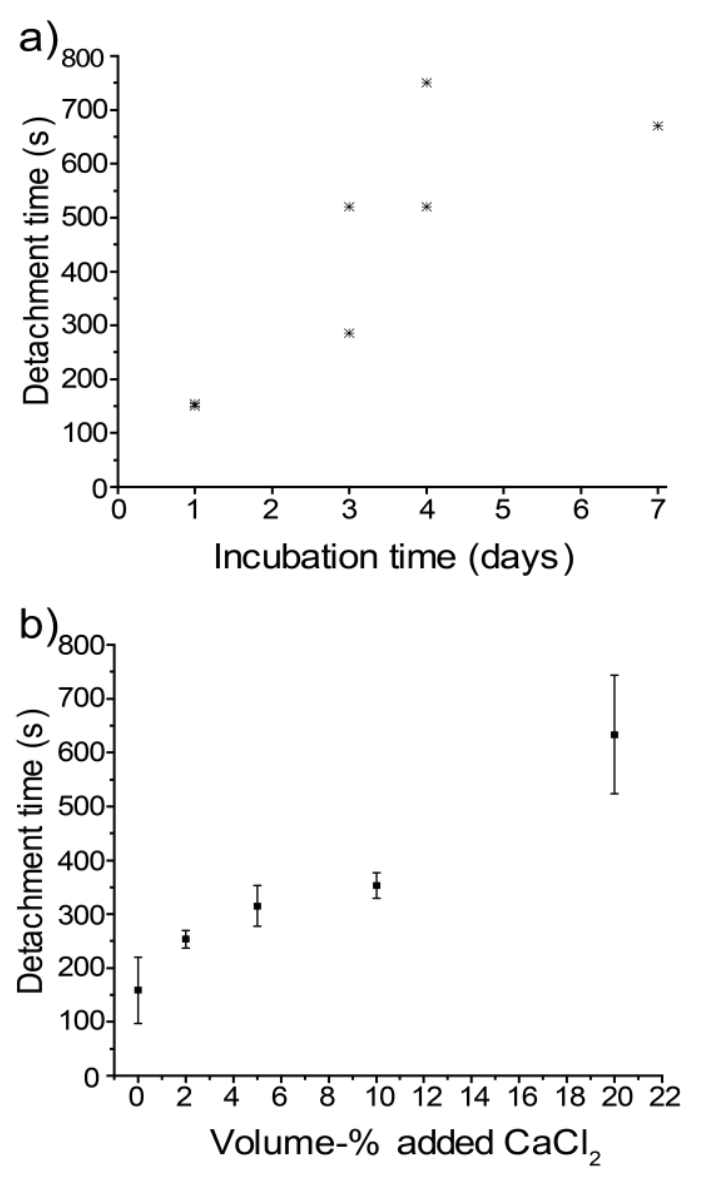

Figure 5. Influence of calcium ions on the detachment of PEDOT-S:H. In a), PEDOT-S:H barcoated on PEDOT:PSS was incubated in $0.1 \mathrm{M} \mathrm{CaCl}_{2}$ for various times and was then moved to $0.1 \mathrm{M} \mathrm{NaCl}$ and detached using 1.0 V. The experiment was performed in duplicate (each experiment represented by an asterisk). For 7 days incubation, only one film could be detached. In b), $0.1 \mathrm{M} \mathrm{CaCl}_{2}$ was added to $0.1 \mathrm{M} \mathrm{NaCl}$. PEDOT-S:H bar-coated on PEDOT:PSS was detached using 1.0 V. Mean and error bars showing standard deviation are displayed, $\mathrm{n}=3$.

Further we compared the detachment processes in electrolytes including a polyanion (PVPA) or a polycation (pDADMAC) with that found in NaCl. PVPA and pDADMAC are both large 
macromolecules that will not easily enter the PEDOT-S:H film. Cyclic voltammetry revealed that oxidation and reduction was possible regardless of the electrolyte (figure 6b). During oxidation in $\mathrm{NaCl}$ and PVPA a small peak is present around $0.2 \mathrm{~V}$, in pDADMAC this peak is absent. Another noticeable feature is the large reduction peak present only in PVPA. It is clear that during redox-cycling of a PEDOT-S:H film, the electrochemical reactions and accompanying ion movements are somewhat different depending on the electrolyte.
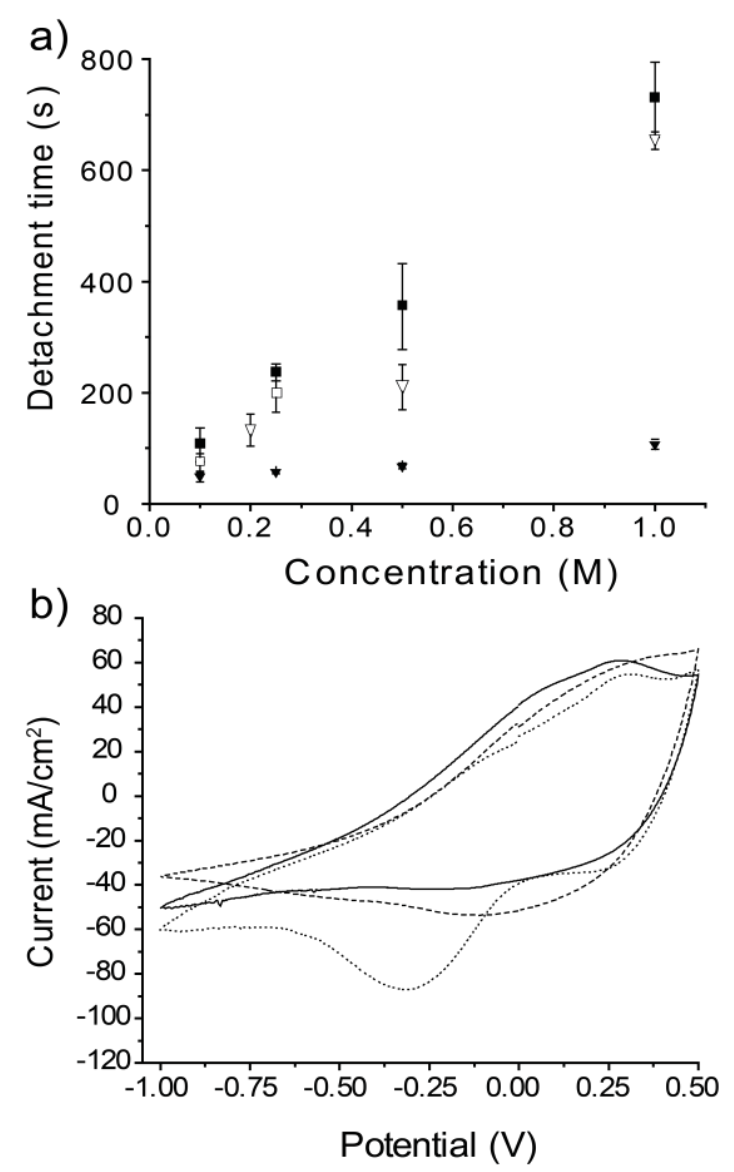

Figure 6. PEDOT-S:H in different electrolytes. a) Detachment of PEDOT-S:H bar coated on PEDOT:PSS using $1.0 \mathrm{~V}$ in different concentrations of $\mathrm{NaCl}$ (black square), PVPA (black triangle), $\mathrm{NaDBS}$ (white square), $\mathrm{Na}_{2} \mathrm{SO}_{4}$ (white triangle), mean and error bars showing standard deviation, $n=3-5$. b) Cyclic voltammetry of PEDOT-S:H films bar coated on PEDOT:PSS in 0.1 
$\mathrm{M}$ of different electrolytes; $\mathrm{NaCl}$ (solid line), pDADMAC (dashed line) and PVPA (dotted line) with a scan rate of $0.02 \mathrm{~V} / \mathrm{s}$.

In $0.1 \mathrm{M}$ pDADMAC no detachment of the PEDOT-S:H films was possible, even though free anions could enter the polymer film. In contrast, in PVPA electrolytes with no free anions, detachment occurred at a relatively rapid rate (figure 6a). This could suggest that the presence of free cations is of importance for the detachment process. That is however unlikely due to the following two reasons. First, free cations are present in $\mathrm{CaCl}_{2}, \mathrm{MgCl}_{2}$ and $\mathrm{FeCl}_{3}$ however no detachment occurred in these electrolytes. Secondly, inclusion of cations is not needed for charge compensation during the oxidation process, as the number of positive (and not negative charges) increases within the film. PEDOT-S:H is a conjugated polyanion and it is therefore possible that pDADMAC will adsorb to the surface of the film and cross-link the outer PEDOT-S:H layers, preventing detachment.

The detachment was considerably faster in PVPA as compared to the other electrolytes (figure 6a). To verify whether this was due to differences in $\mathrm{pH}$ or to the anions present, detachment in $\mathrm{HCl}$ and in PAA (concentrations ranging from $0.1 \mathrm{M}$ to $1.0 \mathrm{M}$ ) was tested. In the $\mathrm{HCl}$ solution the detachment times were similar to those in $\mathrm{NaCl}$, while in PAA the times almost equalled those in PVPA, indicating that it was the anions that influenced the detachment time and not the $\mathrm{pH}$. The very fast detachment times in PVPA can be explained by that the sulphonate side groups of the PEDOT-S:H chains underwent major rearrangement directly at the onset of oxidation in order to counteract positive charges on the polymer backbone introduced via oxidation, as no mobile anions were available. From this, we conclude that the detachment mechanism is a combination of swelling and rearrangement of the polymer chains. 
EQCM-D studies of the detachment in $\mathrm{NaCl}$. The detachment mechanism was also analysed and monitored in more detail using EQCM-D, thus providing a comparison of the change in mass with a simultaneously recorded amperogram. In addition, $\Delta D$ results gives an estimate of the energy dissipation characteristics of the adsorbed mass, with respect to the overall $\Delta f$ and offers vital information regarding both the kinetics and viscoelastic properties of the polymer film. Evaluating the resulting $\Delta D$ and $\Delta f$ values gives a profile of the effective hydrodynamic nature of the deposited polymer film. A lower $\Delta D$ value indicates a less hydrated or "stiffer" polymer layer.

Figure 3 shows the potentiostatic EQCM-D measurements and the corresponding change in frequency and dissipation. A substantial degradation and loss of material from the substrate electrode set in at around $600 \mathrm{~s}, 0.7 \mathrm{~V}(\mathrm{vs} . \mathrm{Ag} / \mathrm{AgCl})$ and the detachment was complete around $60 \mathrm{~s}$ seconds after applying the final voltage step. This relatively faster detachment time found in the EQCM-D experiments is most likely due to the poorer adhesion between PEDOT-S:H and the gold crystals. The mass of the film was correlated with the charge transferred to and from the film. It is evident that the mass increased with the amount of charge transferred to the film and then decreased again as the charge escaped (figure 3b). The transferred charge to the film corresponds to oxidation of PEDOT-S:H and the increase in mass indicates charge compensation, at least in part, by inclusion of $\mathrm{Cl}^{-}$ions. If charge compensation instead would occur through an increased degree of self-doping, there should be an undetectable mass decrease through expulsion of protons.

Applying a constant electronic potential caused an increase in mass accumulated within the polymer film, which was measured by a non-linear frequency decrease and an increase in dissipation. We then used the Sauerbrey model, discussed further in the supporting information, 
to calculate $M_{\mathrm{eq}}$ (table 1$)$ from the change in mass $(\Delta \mathrm{m})$ and the charge transferred to the film $(Q)$. Assuming that this change in mass was entirely due to inclusion of $\mathrm{Cl}^{-}$ions, the corresponding increase in ions included in the film equals more than $1 \mathrm{~mol}$ of $\mathrm{Cl}^{-}$ions $(M=35.5$ $\left.\mathrm{g} \mathrm{mol}^{-1}\right)$. The resulting mass data $\left(M=62 \pm 5 \mathrm{~g} \mathrm{~mol}^{-1}\right)$ instead suggested intercalation of both $\mathrm{Na}^{+}$and $\mathrm{Cl}^{-}$ions in their hydrated state $\left(M_{\mathrm{NaCl}}=60 \mathrm{~g} \mathrm{~mol}^{-1}\right)$ into the film. In general, these results confirmed that the charge neutralization was achieved not only by the expected uptake of chloride ions but also with the addition of sodium to compensate the negatively charged sulphonate chains.

Table 1. Calculations of $M_{\text {eq }}$ from EQCM-D data, using either the Sauerbrey or the Voigt model.

\begin{tabular}{|c|c|c|c|c|c|}
\hline $\begin{array}{l}\text { Voltage } \\
\text { (V) }\end{array}$ & $\begin{array}{l}\text { Charge } \\
(\mathrm{A} \cdot \mathrm{s})\end{array}$ & $\begin{array}{l}\Delta \mathrm{m} \\
\text { Sauerbrey } \\
(\mu \mathrm{g})\end{array}$ & $\begin{array}{l}M_{\mathrm{eq}} \\
\text { Sauerbrey } \\
\left(\mathrm{g} \mathrm{mol}^{-1} \mathrm{e}^{-1}\right)\end{array}$ & $\begin{array}{l}\Delta \mathrm{m} \\
\text { Voigt } \\
(\mu \mathrm{g})\end{array}$ & $\begin{array}{l}M_{\mathrm{eq}} \\
\text { Voigt } \\
\left(\mathrm{g} \mathrm{mol}^{-1} \mathrm{e}^{-1}\right)\end{array}$ \\
\hline 0.3 & 1.32 & 0.78 & 57 & 0.43 & 31.35 \\
\hline 0.4 & 2.04 & 1.13 & 53.5 & 0.62 & 29.4 \\
\hline 0.5 & 3.06 & 1.89 & 59.6 & 1.04 & 32.8 \\
\hline 0.6 & 5.58 & 3.9 & 67.4 & 2.15 & 37.1 \\
\hline 0.7 & - & 0.96 & - & 0.53 & - \\
\hline
\end{tabular}

The dry mass of PEDOT-S:H on the substrate electrode was $80 \mu \mathrm{g}$ and we assumed a selfdoping level of PEDOT-S:H of $25-30 \%$ based on previous studies. ${ }^{15,16,40}$ If we also assume that all incorporated ions, given by the total mass increase, were involved in doping the resulting doping level reached 50-55\% before detachment occurred. Clearly this was not the case since such high doping level is typically not reached in conjugated systems. The resulting observed mass increase must then contain a large fraction of coupled water. There are different reasons for 
the measured excess increase of the mass of the film. One reason is that a PEDOT-S film may show viscoelastic effects ${ }^{41}$ that suppresses the measured oscillation frequency. In the case of a viscoelastic film, the mass of the adlayer may be either overestimated or underestimated by the Sauerbrey model, depending on the mechanical or elastic properties of the film. Since the $f$ and $D$ shifts elicited by the intercalation of ions were considerable in this case, both in their magnitude as well as the irregular nature of their time-dependent profile we tried to employ the Voigt model to determine the overall hydrated mass intercalated in the film. The output from the Voigt modeling and the Sauerbrey relation is shown in Figure 3e, illustrating changes in mass vs. time for the film upon electrochemical oxidation. Considering the complex nature of the physical processes involved during oxidation of the film (first mass increase and then detachment of the film from the gold surface) the agreement between the measured data and the Voigt model must be regarded as good. The best fit for the polymer films was obtained for a film represented by specific input parameters that provides the best data fit for the layer density $\left(1100 \mathrm{~kg} / \mathrm{m}^{3}\right)$, fluid density $\left(1000 \mathrm{~kg} / \mathrm{m}^{3}\right)$, layer viscosity $\left(1^{-5} \leq 1^{-2} \mathrm{~kg} / \mathrm{ms}\right)$, layer shear modulus $\left(1^{3} \leq 1^{7} \mathrm{~Pa}\right)$, and mass $\left(1^{3} \leq 1^{6} \mathrm{ng} / \mathrm{cm}^{2}\right)$. Since the first overtone $(\mathrm{n}=1)$ showed the clearest respons to increases in mass it was included in the model. The first, third and fifth overtones were used for all modeling calculations.

However, in order for the Voigt model and the included parameters to be physically relevant we limited the input data from the start of the experiment to the beginning of detachment (0-600 s). Including data after $600 \mathrm{~s}$ to the Voigt model severely changed the output vector parameters that couldn't be analyzed. The significant increase in both $\Delta D$ and $\Delta f$ after 600 seconds suggest that detachment occurs through softening of the film (increase in $\Delta D$ ) accompanied with almost a linear loss of material between 600-670 s (increase in $\Delta f$ ). 
The modulated increase in mass of the film (table 1, based on the derived parameters above) instead suggested a $45 \%$ decrease of the incorporated mass in total due to viscoelastic contributions from coupled water and electrolyte. Adjusting the mole fraction of incorporated $\mathrm{NaCl}$ to the Voigt-modulated mass, we found an increase in the doping level during oxidation of around $20 \%$, resulting in a final doping level of 37-42\% during oxidation of the PEDOT-S:H film. The EQCM-D results clearly show that in addition to self-doping also inclusion of anions from the electrolyte occurs. The electrode reaction mechanism can be described as:

$$
\left[\mathrm{PEDOT}^{0.25+}-\left(\mathrm{SO}_{3}\right)^{0.25-}\right]-\mathrm{ne}^{-}+\mathrm{nCl}^{-}=\left[\mathrm{PEDOT}^{(0.25+0.12)^{+}}-\left(\mathrm{SO}_{3}\right)^{0.25-}(\mathrm{Cl})^{0.12-}\right]
$$

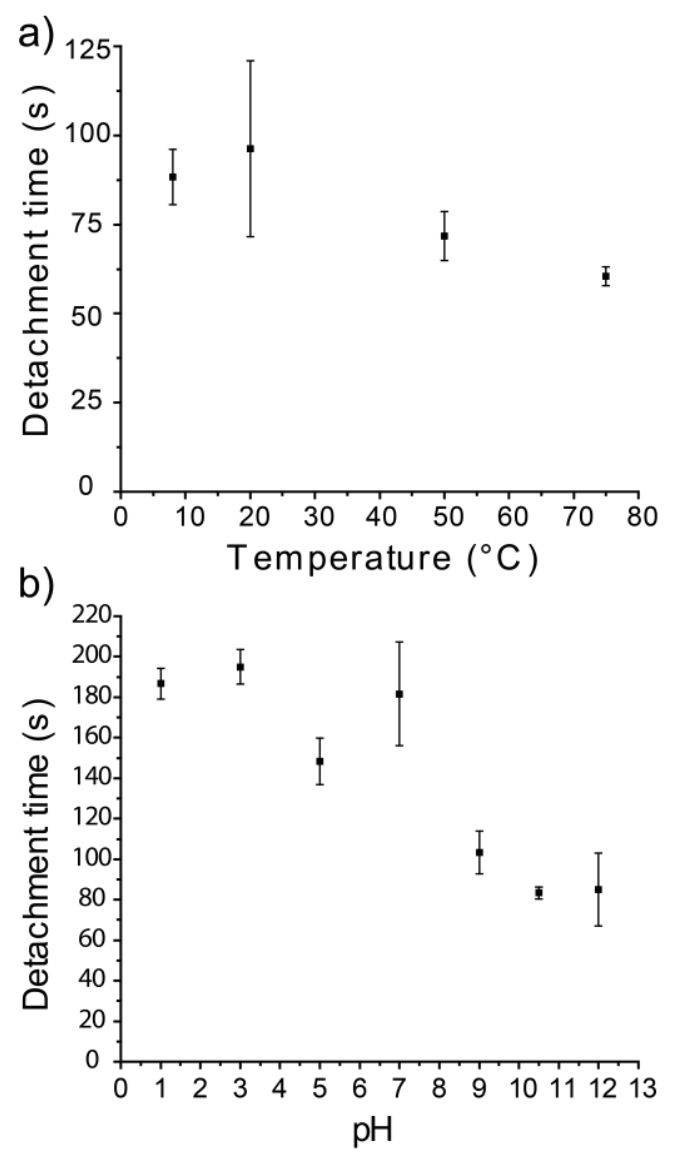

Figure 7. Effect of electrolyte temperature (a) and $\mathrm{pH}$ (b) on detachment time of bar coated PEDOT-S:H on PEDOT:PSS. Detachment with $1.0 \mathrm{~V}$ in $0.1 \mathrm{M} \mathrm{NaCl}$, mean and error bars showing standard deviation, $\mathrm{n}=3$. 
Variations in detachment time. As described above, both the type and concentration of electrolyte will influence the detachment time. Previously we have shown that when we increased the magnitude of the positive potential (vs. $\mathrm{Ag} / \mathrm{AgCl}$ ) applied to the film the detachment time, in $0.1 \mathrm{M} \mathrm{NaCl}$, decreased. ${ }^{20}$ In addition we now evaluated the influence of temperature and $\mathrm{pH}$ of the electrolyte on the detachment time. The effect of heating or cooling the electrolyte was tested in $0.1 \mathrm{M} \mathrm{NaCl}$. We found that heating decreased the detachment time (figure 7a). This was possibly due to faster dynamics of the polymer chains as well as faster swelling due to an increased ion diffusion rate. Also, using $0.1 \mathrm{M} \mathrm{NaCl}$ as electrolyte, the influence of $\mathrm{pH}$ on the detachment time was tested. $\mathrm{HCl}$ or $\mathrm{NaOH}$ was added to the electrolyte to establish the desired $\mathrm{pH}$ values. The detachment times decreased at basic $\mathrm{pH}$ (figure $7 \mathrm{~b}$ ). As mentioned above, PEDOT-S:H is reduced at basic $\mathrm{pH}$ and we assume that this reduction destabilized the polymer film and facilitated faster detachment.

Substrate for detachment. To enable detachment, a conducting layer is needed underneath the PEDOT-S:H film. We have successfully used gold, ITO and other conducting polymer films as substrates for detachment. Without the conducting layer, cracks formed in the PEDOT-S:H film during the oxidation process will terminate conductivity and stop the oxidation from propagating throughout the film, thus hindering detachment. The importance of having a conducting substrate underneath the polymer film is clear from the results in figure 8 . Here, a PEDOT-S:H film was coated on a patterned substrate with individually addressable electrodes separated by non-conducting lines. Even though the PEDOT-S:H film itself was not patterned, it detached only from positively addressed electrodes. The redox changes in the polymer can easily be monitored by recording its electrochromic behaviour, in particular this is easy for the reduced state of PEDOT-S:H that turns dark blue. It was evident that the oxidation (or reduction) did not 
spread from the addressed electrodes through the entire PEDOT-S:H film. This indicates poor lateral conductivity in the wet state as well as anisotrophic properties. ${ }^{50,51}$ The fact that only certain specific parts of a uniform film can be selectively removed might be used as a patterning technique in the future as well as achieving area selective addressing of detachment of a thin film.
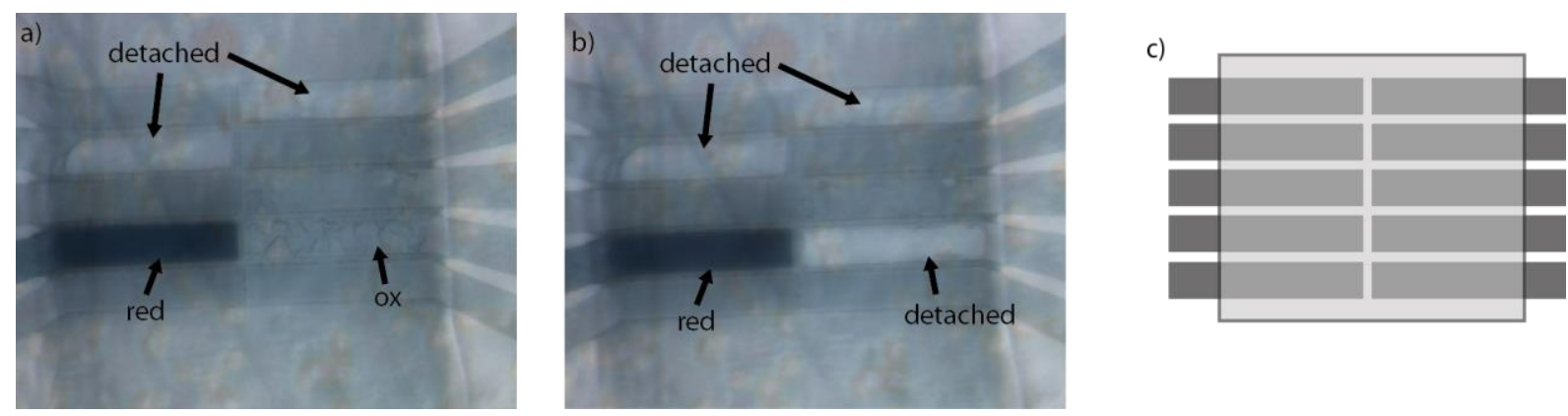

Figure 8. Microscope images of PEDOT-S:H inkjet printed on patterned PEDOT:PSS foil. In a), a potential was applied between the fourth pair of electrodes, reducing PEDOT-S:H on top of the left one and oxidizing PEDOT-S:H on top of the right one. The oxidized PEDOT-S:H is starting to crack and fold. In a) and b), PEDOT-S:H on top of one electrode in the first and second pair has already been detached. In b) PEDOT-S:H that is oxidized in a) has detached. In c), a schematic illustration of the setup is shown. The dark grey lines represent the patterned PEDOT:PSS electrodes and the lighter grey area PEDOT-S:H, that has been inkjet printed on top. The space between the electrodes is $10 \mu \mathrm{m}$, and the line width of the electrodes is $50 \mu \mathrm{m}$.

\section{Conclusions}

We have investigated the detachment mechanism of thin films of the water-soluble and selfdoped conjugated polyelectrolyte PEDOT-S:H. PEDOT-S:H films have been coated on top of underlying electrode substrates. When oxidizing the PEDOT-S:H film it detached from the underlying substrate. A high enough $(>0.05 \mathrm{M})$ concentration of ions in the electrolyte was found to be of major importance in order to maintain a desired stability of the film prior to oxidation. If 
the concentration was lower the film dissolved spontaneously due to inflow of water. Upon submersion in an aqueous electrolyte, the polymer film swelled due to uptake of water and solvated ions.

Upon oxidation, the swelling of the polymer film increased further before it finally detached. We found that the detachment was influenced by several crucial parameters. Increased temperature and basic $\mathrm{pH}$ values both decreased the detachment time. Moreover, di- or trivalent cations seemed to cross-link the PEDOT-S:H chains, thus preventing detachment. When increasing the electrolyte concentration, the detachment time increased, most likely due to less polymer swelling in turn caused by a decrease in the concentration differences between polymer and electrolyte. Upon oxidation, the resulting positive charges on the polymer backbone can be balanced either by anions from the electrolyte or by the negative sulphonate side groups of the polymer itself (more self-doping), or by both in combination. In the case of a polyanion electrolyte, no free anions are available. Detachment in this case was found to be faster than in electrolytes including small-sized anions. This indicates that it is the rearrangement of the polymer side chains that drives the actual detachment process. We therefore suggest that the detachment mechanism takes place in three parts, which was also supported by EQCM-D studies; first swelling due to inflow of water and electrolyte regardless of any applied potential occur, secondly an inflow of mobile anions (if any) will increase the swelling even further, thirdly the polymer chains rearrange to allow more self-doping. The rearrangement in turn leads to cracking and detachment of the entire film. In electrolytes with mobile small-sized anions the first part of oxidation includes diffusion of the anions into the polymer film to counter-balance positive charges, however rearrangement will eventually take place, leading to detachment. 
We have reported an in depth study of the detachment mechanism of PEDOT-S:H. This material exhibits excellent detachment characteristics above a threshold potential of $+0.7 \mathrm{~V}$ vs. a $\mathrm{Ag} / \mathrm{AgCl}$ reference electrode. We believe that electronic control of detachment is of generic interest in areas related to active control over the release of cells, tissues, and drugs and also for more technological areas, such as the electronic glue in packaging, assembly and construction technology.

\section{ASSOCIATED CONTENT}

Supporting information: EQCM-D background, detachment methods, EQCM-D methods, AFM methods, patterning methods, ion movements figure. This material is available free of charge via the Internet at http://pubs.acs.org.

\section{AUTHOR INFORMATION}

\section{Corresponding Author}

*E-mail: magnus.berggren@liu.se

\section{Notes}

The authors declare no competing financial interests.

\section{ACKNOWLEDGMENT}

This work is conducted within the OBOE-IFH Environment, supported by VINNOVA 201000507. It is also supported by KAW and Önnesjö Foundation and the authors also acknowledge financial support from the Swedish Government Strategic Research Area in Materials Science on Functional Materials at Linköping University (Faculty Grant SFO-Mat-LiU \# 2009-00971).

\section{REFERENCES}


(1) Berggren, M.; Richter-Dahlfors, A. Organic Bioelectronics. Adv. Mater. 2007, 19, 3201-3213.

(2) Guimard, N. K.; Gomez, N.; Schmidt, C. E. Conducting polymers in biomedical engineering. Prog. Polym. Sci. 2007, 32, 876-921.

(3) Asplund, M.; Thaning, E.; Lundberg, J.; Sandberg-Nordqvist, A. C.; Kostyszyn, B.; Inganäs, O.; Holst, von, H. Toxicity evaluation of PEDOT/biomolecular composites intended for neural communication electrodes. Biomed. Mater. 2009, 4, 1-12.

(4) George, P. M.; Lyckman, A. W.; LaVan, D. A.; Hegde, A.; Leung, Y.; Avasare, R.; Testa, C.; Alexander, P. M.; Langer, R.; Sur, M. Fabrication and biocompatibility of polypyrrole implants suitable for neural prosthetics. Biomaterials 2005, 26, 3511-3519.

(5) Abidian, M. R.; Kim, D. H.; Martin, D. C. Conducting Polymer Nanotubes for Controlled Drug Release. Adv. Mater. 2006, 18, 405-409.

(6) Pernaut, J.-M.; Reynolds, J. R. Use of Conducting Electroactive Polymers for Drug Delivery and Sensing of Bioactive Molecules. A Redox Chemistry Approach. J. Phys. Chem. B 2000, 104, 4080-4090.

(7) Cui, X.; Wiler, J. A.; Dzaman, M.; Altschuler, R. A.; Martin, D. C. In vivo studies of polypyrrole/peptide coated neural probes. Biomaterials 2003, 24, 777-787.

(8) Ludwig, K. A.; Uram, J. D.; Yang, J.; Martin, D. C.; Kipke, D. R. Chronic neural recordings using silicon microelectrode arrays electrochemically deposited with a poly(3,4-ethylenedioxythiophene) (PEDOT) film. J. Neural Eng. 2006, 3, 59-70.

(9) Yamoto, H.; Ohwa, M.; Wernet, W. Stability of polypyrrole and poly(3,4-ethylenedioxythiophene) for biosensor application. J. Electroanal. Chem. 1995, 397, 163-170.

(10) Groenendaal, L. B.; Jonas, F.; Freitag, D.; Pielartzik, H.; Reynolds, J. R. Poly(3,4ethylenedioxythiophene) and Its Derivatives: Past, Present, and Future. Adv. Mater. 2000, 12, 481-494. 
(11) Hamedi, M.; Herland, A.; Karlsson, R. H.; Inganäs, O. Electrochemical Devices Made From Conducting Nanowire Networks Self-Assembled From Amyloid Fibrils and Alkoxysulfonate PEDOT. Nano Lett. 2008, 8, 1736-1740.

(12) Müller, C.; Jansson, R.; Elfwing, A.; Askarieh, G.; Karlsson, R.; Hamedi, M.; Rising, A.; Johansson, J.; Inganäs, O.; Hedhammar, M. Functionalisation of recombinant spider silk with conjugated polyelectrolytes. J. Mater. Chem. 2011, 21, 2909

(13) Hamedi, M.; Elfwing, A.; Gabrielsson, R.; Inganäs, O. Electronic Polymers and DNA SelfAssembled in Nanowire Transistors. Small 2013, 9, 363-368.

(14) Patil, A. O.; Ikenoue, Y.; Basescu, N.; Colaneri, N.; Chen, J.; Wudl, F.; Heeger, A. J. Self-doped conducting polymers. Synthetic Met. 1987, 20, 151-159.

(15) Patil, A. O.; Ikenoue, Y.; Wudl, F.; Heeger, A. J. Water-soluble conducting polymers. J. Am. Chem. Soc. 1987, 109, 1858-1859.

(16) Cutler, C. A.; Bouguettaya, M.; Kang, T.-S.; Reynolds, J. R. Alkoxysulfonate-Functionalized PEDOT Polyelectrolyte Multilayer Films: Electrochromic and Hole Transport Materials. Macromolecules 2005, 38, 3068-3074.

(17) Stéphan, O.; Schottland, P.; Le Gall, P.-Y.; Chevrot, C.; Mariet, C.; Carrier, M. Electrochemical behaviour of 3,4-ethylenedioxythiophene functionalized by a sulphonate group. Application to the preparation of poly(3,4-ethylenedioxythiophene) having permanent cation-exchange properties. $J$. Electroanal. Chem. 1998, 443, 217-226.

(18) Karlsson, R. H.; Herland, A.; Hamedi, M.; Wigenius, J. A.; Åslund, A.; Liu, X.; Fahlman, M.; Inganäs, O.; Konradsson, P. Iron-Catalyzed Polymerization of Alkoxysulfonate-Functionalized 3,4Ethylenedioxythiophene Gives Water-Soluble Poly(3,4-ethylenedioxythiophene) of High Conductivity. Chem. Mater. 2009, 21, 1815-1821. 
(19) Zotti, G.; Zecchin, S.; Schiavon, G.; Groenendaal, L. B. Electrochemical and Chemical Synthesis and Characterization of Sulfonated Poly(3,4-Ethylenedioxythiophene): a Novel Water-Soluble and Highly Conductive Conjugated Oligomer. Macromol. Chem. Phys. 2002, 203, 1958-1964.

(20) Persson, K. M.; Karlsson, R.; Svennersten, K.; Löffler, S.; Jager, E. W. H.; Richter-Dahlfors, A.; Konradsson, P.; Berggren, M. Electronic Control of Cell Detachment Using a Self-Doped Conducting Polymer. Adv. Mater. 2011, 23, 4403-4408.

(21) Huang, H.-L.; Hsing, H.-W.; Lai, T.-C.; Chen, Y.-W.; Lee, T.-R.; Chan, H.-T.; Lyu, P.-C.; Wu, C.-L.; Lu, Y.-C.; Lin, S.-T.; et al. Trypsin-Induced Proteome Alteration During Cell Subculture in Mammalian Cells. J Biomed Sci 2010, 17, 36.

(22) Gao, J.; Pang, Z.; Chen, Q.; Zheng, L.-R. Interactive Packaging Solutions Based on RFID Technology and Controlled Delamination Material. Int. IEEE Conf. RFID 2010, 158-165.

(23) Liu, Y.; Gan, Q.; Baig, S.; Smela, E. Improving PPy Adhesion by Surface Roughening. J. Phys. Chem. C 2007, 111, 11329-11338.

(24) Mukoyama, I.; Aoki, K.; Chen, J. Electrochemical Dissolution of Polythiophene Films. J. Electroanal. Chem. 2002, 531, 133-139.

(25) Warren, L. F.; Anderson, D. P. Polypyrrole Films from Aqueous Electrolytes. J. Electrochem. Soc. 1987, 134, 101-105.

(26) Zotti, G.; Zecchin, S.; Schiavon, G.; Louwet, F.; Groenendaal, L.; Crispin, X.; Osikowicz, W.; Salaneck, W.; Fahlman, M. Electrochemical and XPS Studies toward the Role of Monomeric and Polymeric Sulfonate Counterions in the Synthesis, Composition, and Properties of Poly(3,4ethylenedioxythiophene). Macromolecules 2003, 36, 3337-3344. 
(27) Gandhi, M. R.; Murray, P.; Spinks, G. M.; Wallace, G. G. Mechanism of electromechanical actuation in polypyrrole. Synthetic Met. 1995, 73, 247-256.

(28) Wang, X.; Smela, E. Color and Volume Change in PPy(DBS). J. Phys. Chem. C 2009, 113, 359368.

(29) Aygun, A.; Buthker, J. W.; Stephenson, L. D.; Kumar, A.; Mahle, T. K.; Gewirth, A. A. Journal of Electroanalytical Chemistry. J. Electroanal. Chem. 2012, 684, 47-52.

(30) Smela, E.; Gadegaard, N. Volume Change in Polypyrrole Studied by Atomic Force Microscopy. J. Phys. Chem. B 2001, 105, 9395-9405.

(31) Carlberg, C.; Chen, X.; Inganäs, O. Ionic transport and electronic structure in poly(3,4ethylenedioxythiophene). Solid State Ionics 1996, 85, 73-78.

(32) Chen, X.; Xing, K.-Z.; Inganäs, O. Electrochemically Induced Volume Changes in Poly(3,4ethylenedioxythiophene). Chem. Mater. 1996, 8, 2439-2443.

(33) Jager, E. W. H.; Smela, E.; Inganäs, O. Microfabricating Conjugated Polymer Actuators. Science 2000, 290, 1540-1545.

(34) Gaihre, B.; Alici, G.; Spinks, G. M.; Cairney, J. M. Sensors and Actuators a: Physical. Sens. Actuat. A. 2011, 165, 321-328.

(35) Okuzaki, H.; Suzuki, H.; Ito, T. Electrically Driven PEDOT/PSS Actuators. Synthetic Met. 2009, $159,2233-2236$.

(36) Pei, Q.; Inganäs, O. Electrochemical applications of the bending beam method. 2. Electroshrinking and slow relaxation in polypyrrole. $J$. Phys. Chem. 1993, 97, 6034-6041.

(37) Pigani, L.; Heras, A.; Colina, Á.; Seeber, R.; López-Palacios, J. Electropolymerisation of 3,4ethylenedioxythiophene in aqueous solutions. Electrochem. Commun. 2004, 6, 1192-1198. 
(38) Bruckenstein, S.; Brzezinska, K.; Hillman, A. R. EQCM studies of polypyrrole films. 1. Exposure to aqueous sodium tosylate solutions under thermodynamically permselective conditions. Electrochim. Acta 2000, 45, 3801-3811.

(39) Nagaraju, D. H.; Rebis, T.; Gabrielsson, R.; Elfwing, A.; Milczarek, G.; Inganäs, O. Charge Storage Capacity of Renewable Biopolymer/Conjugated Polymer Interpenetrating Networks Enhanced by Electroactive Dopants. Adv. Energy Mater. 2013, 4.

(40) Yi, P.; Chen, K. L. Release Kinetics of Multiwalled Carbon Nanotubes Deposited on Silica Surfaces: Quartz Crystal Microbalance with Dissipation (QCM-D) Measurements and Modeling. Environ. Sci. Technol. 2014, 48, 4406-4413.

(41) Chowdhury, I.; Duch, M. C.; Mansukhani, N. D.; Hersam, M. C.; Bouchard, D. Deposition and Release of Graphene Oxide Nanomaterials Using a Quartz Crystal Microbalance. Environ. Sci. Technol. 2014, 48, 961-969.

(42) Delcroix, M. F.; Demoustier-Champagne, S.; Dupont-Gillain, C. C. Quartz Crystal Microbalance Study of Ionic Strength and pH-Dependent Polymer Conformation and Protein Adsorption/Desorption on PAA, PEO, and Mixed PEO/PAA Brushes. Langmuir 2014, 30, 268-277.

(43) Benedetti, T. M.; Torresi, R. M. Rheological Changes and Kinetics of Water Uptake by Poly(Ionic Liquid)-Based Thin Films. Langmuir 2013, 29, 15589-15595.

(44) Pei, Q.; Inganäs, O. Electrochemical applications of the bending beam method. 1. Mass transport and volume changes in polypyrrole during redox. J. Phys. Chem. 1992, 96, 10507-10514.

(45) Wang, X.; Shapiro, B.; Smela, E. Development of a Model for Charge Transport in Conjugated Polymers. J. Phys. Chem. C 2009, 113, 382-401. 
(46) Bay, L.; Jacobsen, T.; Skaarup, S.; West, K. Mechanism of Actuation in Conducting Polymers: Osmotic Expansion. J. Phys. Chem. B 2001, 105, 8492-8497.

(47) Velmurugu, Y.; Skaarup, S. Ion and Solvent Transport in Polypyrrole: Experimental Test of Osmotic Model. Ionics 2005, 11, 370-374.

(48) Ghosh, S.; Inganäs, O. Conducting Polymer Hydrogels as 3D Electrodes: Applications for Supercapacitors. Adv. Mater. 1999, 1214-1218.

(49) Jafeen, M. J. M.; Careem, M. A.; Skaarup, S. Speed and strain of polypyrrole actuators: dependence on cation hydration number. Ionics 2009, 16, 1-6.

(50) van de Ruit, K.; Cohen, R. I.; Bollen, D.; van Mol, T.; Yerushalmi-Rozen, R.; Janssen, R. A. J.; Kemerink, M. Quasi-One Dimensional in-Plane Conductivity in Filamentary Films of PEDOT:PSS. Adv. Funct. Mater. 2013, 23, 5778-5786.

(51) van de Ruit, K.; Katsouras, I.; Bollen, D.; van Mol, T.; Janssen, R. A. J.; de Leeuw, D. M.; Kemerink, M. The Curious Out-of-Plane Conductivity of PEDOT:PSS. Adv. Funct. Mater. 2013, 23, $5787-5793$.

\section{TOC graphic}

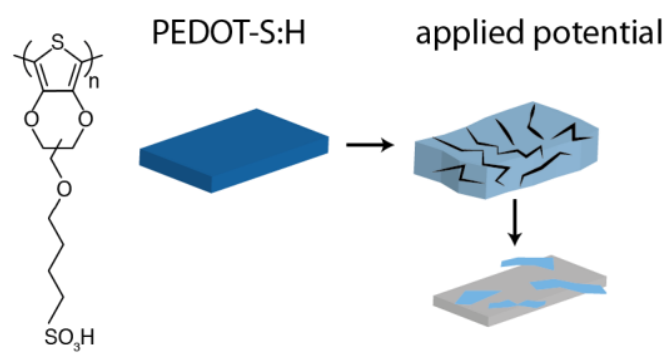

